\title{
QUESTIONS DECEMBER 2013
}

CPD questionnaires must be completed online via www.cpdjournals.co.za

After submission you can check the answers and print your certificate.

True (A) or false (B):

A cost-effective strategy for primary prevention of acute rheumatic fever and rheumatic heart disease in children with pharyngitis

1. Cost-effectiveness modelling shows that treating only children with $\geq 2$ symptoms (CDR2+) - on a 3-symptom (presence of enlarged cervical nodes, absence of rhinitis and absence of rash) score - is the most cost-effective for primary prevention.

2. The antibiotic of choice for such treatment is ampicillin.

Blood cultures in sick children

3. A dramatic decline has been noted in the culture of Streptococcus pneumoniae, reflecting the effectiveness of the national pneumococcal immunisation programme and the declining paediatric HIV incidence.

4. The Integrated Management of Childhood Illness (IMCI) programme advises that any seriously ill child requiring urgent referral to hospital should receive intramuscular ceftriaxone prior to transfer.

5. The IMCI programme accounts for the high contamination rates ( 5 - 7\%) by coagulase-negative staphylococci.

Management of acute fever in children

6. While most fevers are due to a self-limiting viral infection, serious bacterial infections are more common in young children.

7. Fever in a child aged $<3$ months should be investigated thoroughly and referred to specialist care if the source of the fever cannot be found.

8. Oral and rectal routes are recommend to measure body temperature in a child.

9. Empirical antibiotics for possible occult bacteraemia in children aged $>3$ months does not confer any significant advantage.

10. As antipyretics, both paracetamol and ibuprofen are safe and effective for short-term use in children.

11. Antipyretics assist in preventing febrile convulsions.

12. Aspirin should not be used in children younger than 12 years of age because of the risk of Reye's syndrome.
13. Prophylactic administration of antipyretic drugs at the time of vaccination is associated with reduced antibody responses to vaccine antigens.

Combined paediatric liver-kidney transplantation (CPLKT)

14. The chief indication for CPLKT is for rare congenital diseases affecting both liver and kidney (such as primary hyperoxaluria type 1 and autosomal recessive polycystic kidney disease with hepatic fibrosis).

15. Mortality post-transplant is typically as a result of organ rejection.

A comparison of the pharmacokinetics of Aspen Ceftriaxone and Rocephin in community-acquired meningitis

16. The main causes of community-acquired bacterial meningitis in immunocompetent patients are S. pneumoniae, Neisseria meningitidis, Haemophilus influenzae and, less commonly, Listeria monocytogenes.

17. In immunocompromised patients, specifically those with HIV/AIDS, S. pneumoniae remains the most frequently isolated organism.

A follow-up cross sectional study of environmental lead exposure in early childhood in urban South Africa (SA)

18. Of SA children residing in urban areas, $70-80 \%$ have blood levels $\geq 5 \mu \mathrm{g} / \mathrm{dl}$ (exceeding the current reference level of the US Centres for Disease Control).

Paediatric spirometry guideline of the South African Thoracic Society: Part 1

19. There are three types of lung function patterns detected by spirometry testing: obstructive, mixed and restrictive.

20. Because young children have a smaller total lung volume and a larger airway size relative to lung volume than older children and adults, the forced expiration is completed in a shorter time.

\section{INSTRUCTIONS}

1. Read the journal. All the answers will be found there.

2. Go to www.cpdjournals.co.za to answer the questions.

Accreditation number: MDB001/006/01/2013 (Clinical) 